\title{
Modeling of Viscous Fingering based on the numerical solution of the Navier-Stokes Equations in 3D.
}

\author{
Andres Pinilla1, Luis Ramirez ${ }^{1}$, Miguel Asuaje², Nicolas Ratkovich ${ }^{1}$ \\ ${ }^{1}$ University of los Andes \\ CR1\#18A-12, Bogota, Colombia. \\ First.ja.pinilla1368@uniandes.edu.co; la.ramirez15@uniandes.edu.co \\ ${ }^{2}$ Simon Bolivar University \\ Sartenejas, Caracas, Venezuela \\ asuajem@usb.ve
}

\begin{abstract}
The prediction of multiphase flow in porous media is of great interest in several fields like Oil \& Gas, $\mathrm{CO}_{2}$ sequestration, geothermal energy, among others. Subsurface modeling is challenging due to the difficulties in predicting microscopic phenomena like Viscous Fingering that manifest at the macroscale in channellings, affecting displacement efficiency. Viscous Fingering is characterized for causing finger-like patterns that channels the phase with the lowest viscous resistance in the porous media. This phenomenon is the root cause of heavy oil production inefficiency, causing excessive water production, increasing costs, and energy consumption. This study proposes a numerical model based on the complete set of the Navier-Stokes equations in 3D to predict this natural phenomenon. The numerical model, developed using commercial CFD software, is based on an Eulerian-VOF solution to simulate immiscible multiphase flow in porous media in 3D. To verify the numerical model, it was compared against experimental data for oil recovery on three different viscosity ratios, ranging from $10^{1}$ to $10^{3}$. The deviation against the experimental data was below $10 \%$, achieving a successful prediction on the oil recovery while qualitatively predicting Viscous Fingering in $3 \mathrm{D}$ in great detail. Several differences between the studied viscosity ratios were found too. Not only regarding the oil recovery but also noticeable differences between the fingering dynamics were found too. The numerical solution proposed in this study could be helpful for scientists interested in subsurface modeling in great detail, where the prediction of multiphase flow in porous media is of great interest to improve subsurface processes.
\end{abstract}

Keywords: Navier-Stokes, Viscous Fingering, Unstable displacements, Multiphase flow in porous media.

\section{Introduction}

Flow in porous media has been studied for centuries. Henry Darcy proposed one of the most representative mathematical models almost two centuries ago, working for single-phase flows. However, for multiphase flow in porous media, there is not a mathematical solution yet. These displacements are more complex, characterized for having different flow patterns depending on the competition between capillary and viscous forces, ultimately determining the displacement efficiency. Three main flow patterns have been identified for immiscible displacements, stable displacements, capillary Fingering, and Viscous Fingering [1], [2].

Viscous Fingering (VF) is the most inefficient displacement mechanism and occurs when the viscous forces overcome the capillary ones. This condition usually occurs at relatively high flow rates or high viscosity contrasts between the phases. It is characterized for presenting finger-like patterns, which tend to channel the less viscous resistance phase through the more viscous one. Although scientist knew that multiphase flows in porous media were characterized for having an unstable displacement or a non-piston-like interface, like Morris Muskat [3], [4] or Buckley \& Leverett[5] in the mid-1930s and '40s, it was not until the early 1950s that the actual displacement mechanisms were discovered. Engelberts \& Klinkenberg [6] and Hill \& Inst [7] reported VF in separate experimental studies for the first time. To this date, this phenomenon, and multiphase flows in porous media in general, have been extensively studied due to their implications in several fields, especially in subsurface processes.

One of the most affected industries by VF is the oil industry. This phenomenon is the root cause of high water production, which causes the exponential growth in lifting costs [8] and the consequent increase in energy consumption [9]. An alternative to mitigate or prevent this issue is predicting the movement of the fluids near the wellbore to conduct corrective actions. There are several technologies to predict multiphase flow in porous media. For example, solutions based on the 
Diffusion-Limited Aggregation method use a stochastic approach to simulate VF [10]-[14]. Solutions based on the diffusivity equation and black oil models like reservoir simulation codes, which use use a finite volume method using the Representative Elementary Volume approach. Alternatively, solutions are based on the conservation equations of fluid flow. These last ones using solutions based on Lattice-Boltzmann [15]-[17] or Galerkin [18]-[20] methods

In this study, a numerical solution of the Navier-Stokes equations is proposed for simulating VF in great detail in 3D. The CFD commercial software STAR-CCM+ was used, implementing an Eulerian-Volume of Fluid (VOF) solution for simulating the multiphase flow in porous media using a polyhedral unstructured mesh based on the findings of a previous study[21]. The numerical model was validated against experimental data found in the literature [22] of coreflood experiments for three different viscosity ratios, 60,1440, and 10500. The numerical model could predict oil recovery through time and emulate the fingering dynamics with noticeable differences between the three viscosity ratios.

\section{Methodology}

As mentioned before, the numerical model was developed using commercial CFD software. In addition, the simulations were run in a computer with an Inter Xenon E5-2673 processor and 56GB RAM. The numerical model is based on the numerical solution of the conservation equations of fluid flow. The model also assumes that the fluids are incompressible and Newtonian, therefore, numerically solving the complete set of the Navier-Stokes equations in 3D.

The numerical model simulates the coreflood experiments conducted by Doorwar \& Mohanty [22]. Therefore, it uses a $12 \mathrm{in}$ long and $2 \mathrm{in}$ in diameter core sample. The general description of the core dimensions, injection flowrate, and boundary conditions are presented in Fig. 1. Additionally, this figure presents a general view of the spatial discretization used to conduct the numerical experiments. Finally, the displacements in the numerical experiments were carried in the vertical direction, assuming gravity as an external body force acting against the flow direction.

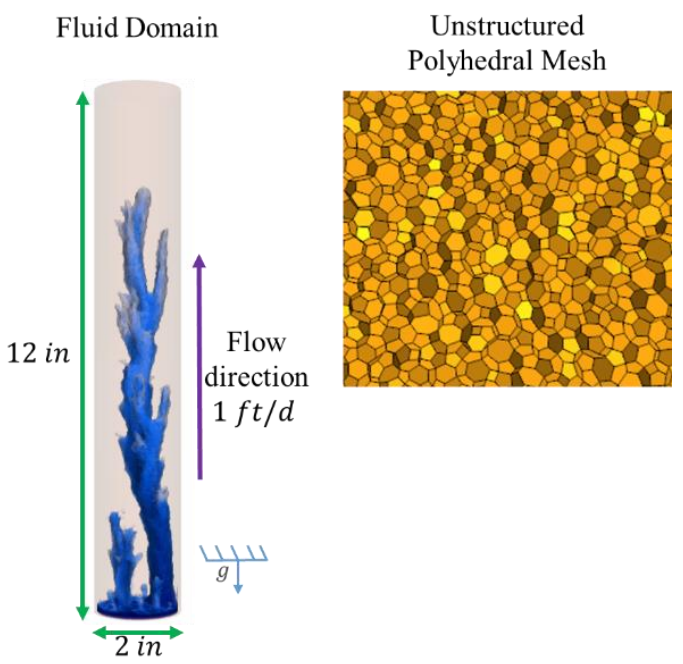

Fig. 1: Description of the core sample used and the spatial discretization implemented in the numerical experiments.

Finally, Table 1 presents the primary fluid and porous physical properties, also adapted from the experiments found in the literature. It must be pointed out that the numerical model considers homogeneous porous properties. Also, the experiments used heavy oil and water as displaced and displacing fluids, respectively. 
Table 1: Fluid and porous physical properties.

\begin{tabular}{|c|c|}
\hline \multicolumn{2}{|l|}{ Fluid Properties } \\
\hline Viscosity ratios $\left(\mu_{r}\right)$ & $\begin{array}{c}60 \\
1440 \\
10500\end{array}$ \\
\hline \multicolumn{2}{|l|}{ Rock Properties } \\
\hline Porosity (\%) & 29 \\
\hline Permeability (d) & 6 \\
\hline Initial water saturation (\%) & 17 \\
\hline
\end{tabular}

\section{Results}

\subsection{Prediction of oil recovery}

One of the most important results of the numerical model is the prediction of oil recovery through time. As mentioned before, experimental data found in the literature was used to verify the numerical model for three different viscosity ratios. Fig. 2 presents the oil recovery profiles obtained with the CFD numerical model, where an absolute mean error below $10 \%$ was found for the three study cases.

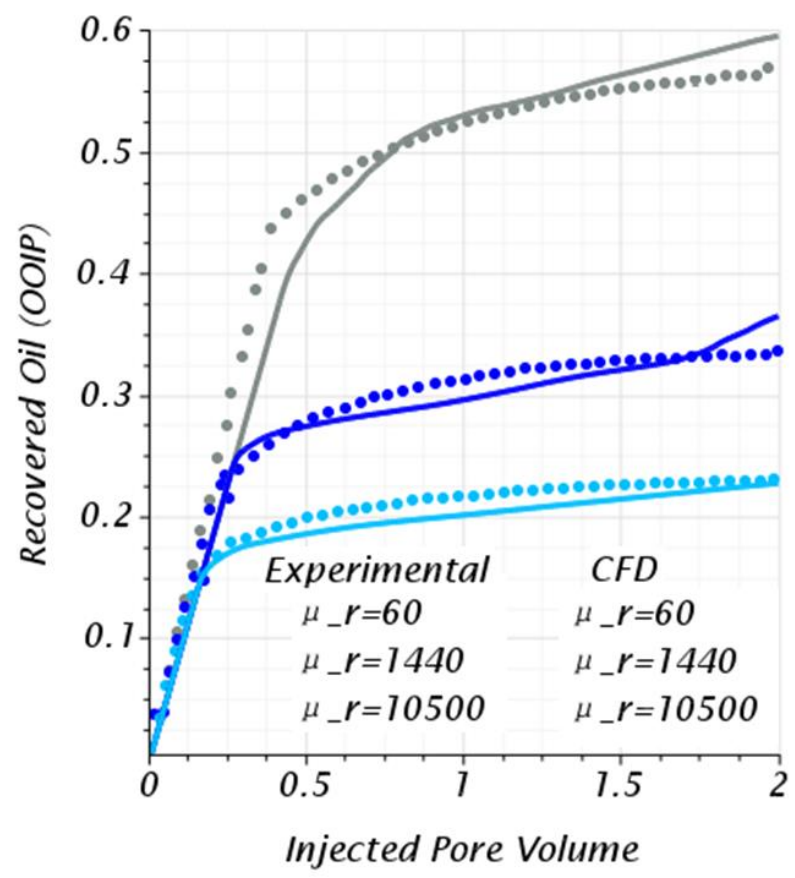

Fig. 2: Description of the core sample used and the spatial discretization implemented in the numerical experiments.

On the one hand, these results show that the numerical model could predict the differences in the oil recovery before the breakthrough event and the breakthrough time between the different viscosity ratios. For example, it is expected that at higher viscosity ratios, the breakthrough time is reduced as a consequence of the higher viscous forces, which worsens the Fingering. In this case, the numerical model accurately predicted a faster breakthrough time for the higher viscosity ratio, $\mu_{r}=10500$, with lower breakthrough times for the other two cases. Also, with a suitable adjustment against the experimental data. The 
breakthrough event is inferred by the change in slope of the oil recovery profiles. The prediction results of the breakthrough time are presented in Table 2, where the model accurately predicted the breakthrough time for the most viscous oils. At the same time, the highest deviation was found for the less viscous oil with a mean absolute error of around $10 \%$

Table 2: Fluid and porous physical properties.

\begin{tabular}{|c|c|c|c|}
\hline Viscosity ratio & $\begin{array}{c}\text { Experimental } \\
\text { (IPV-aprox) }\end{array}$ & $\begin{array}{c}\text { Numerical } \\
\text { (IPV-aprox) }\end{array}$ & $\begin{array}{c}\text { Mean Absolute } \\
\text { error (\%) }\end{array}$ \\
\hline 60 & 0.40 & 0.44 & 10 \\
\hline 1440 & 0.28 & 0.27 & 3.30 \\
\hline 10500 & 0.16 & 0.16 & $<1$ \\
\hline
\end{tabular}

Additionally, the numerical model reproduced the behavior in oil recovery before and after the breakthrough event. For example, it is expected that higher viscous oils have a lower oil recovery, a direct consequence of the Fingering, shown in Fig. 2. Before the breakthrough event, the less viscous oil, i.e., lower viscosity ratio, achieves a higher oil recovery than its counterparts. Also, after the breakthrough, it has a higher slope, reflecting a higher oil recovery. On the contrary, after the breakthrough, the other two cases have a lower slope, indicating that the Fingering is worse, reducing the sweep efficiency and reducing oil recovery. The following subsection will elucidate how Fingering worsens at higher viscosity ratios by showing the 3D dynamics of the Fingering, explaining why oil recovery is reduced.

\subsection{Prediction of Viscous Fingering dynamics}

One of the primary goals of predicting VF is emulating its characteristic features, for example, capturing the finger birth, growth, splitting, side-branching, coalescence, among others. Even more, some of these features become dominant at higher viscosity ratios, indicating the severity of the Fingering. The CFD numerical model could emulate the dynamics of $\mathrm{VF}$ and the differences between the three studied viscosity ratios. Table 3 presents some 3D renders at different times, where the Fingering is successfully emulated, and several differences between the three viscosity ratios are noticed.

For example, it was noticed that water tends to displace around the core walls for the lowest viscosity ratio. On the contrary, for the highest viscosity ratios, water displaced through the middle. Also, the lowest viscosity ratio tends to have rounder fingers with no noticeable side branching or tip-splitting. This feature makes the displacement more stable, explaining why a higher sweep efficiency can be reached, achieving a higher oil recovery before and after the breakthrough event.

For the viscosity ratio of 1140 , the fingering dynamics change dramatically. In this case, the displacement is more unstable, and the Fingering worsens. Moreover, side-branching becomes dominant, as shown in Table 3. However, these branches do not grow and become stuck relatively fast. New characteristic features of VF appear too. For example, the blocking mechanisms of the dominant fingers over the smaller ones, which aborted their displacement at the very beginning. Finally, the sweep efficiency gets reduced as narrower fingers are dominant. They are sharper and not rounded and wider, as in the lower viscosity ratio case.

For the last case, the viscosity ratio of 10500 , the severity of the Fingering worsens noticeably. For example, a single dominant and narrow finger characterize the displacement. The blocking mechanism is a dominant feature from the beginning of the displacement, where water channels exclusively through this dominant finger. Because of this feature, the sweep efficiency is remarkably lower compared to the other two cases. Finally, it can be noticed that the amount of oil left behind, showed as transparent in the renders of Table 3. 
Table 3: Viscous Fingering dynamics at different times for the different viscosity ratios

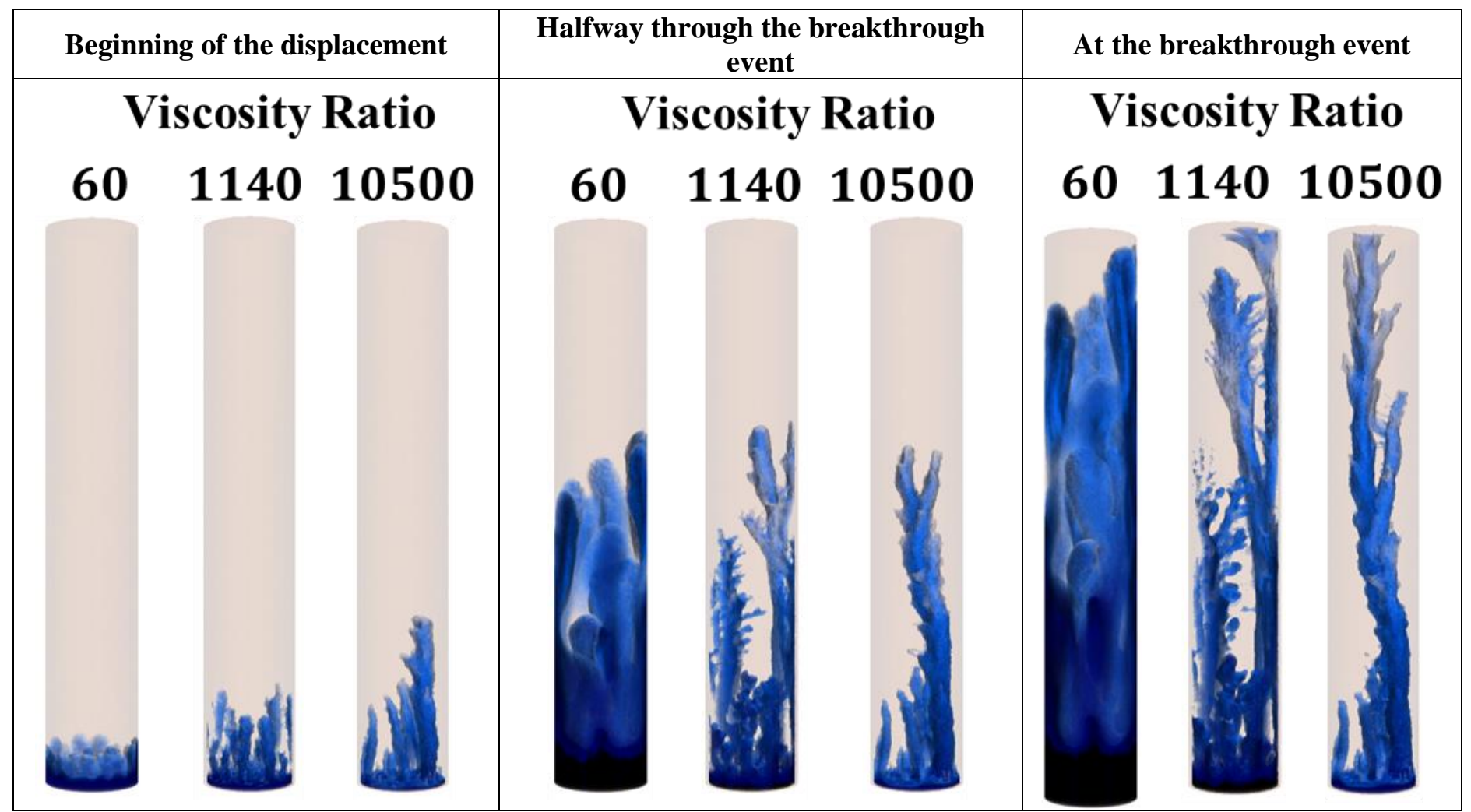

\section{Conclusion}

A numerical model based on the complete set of the Navier-Stokes equations capable of emulating VF was successfully developed. The model was validated against experimental data for three different viscosity ratios with a mean absolute error below $10 \%$. The prediction of the breakthrough time and oil recovery through time was successfully achieved.

Solutions based on the Navier-Stokes equations are capable of emulating the fingering dynamics. Moreover, different viscosity ratios can predict dominant features. For example, round fingers for low viscosity ratios, dominant side branching at viscosity ratios up to $10^{2}$, or dominant tip splitting and one dominant finger at viscosity ratios up to $10^{3}$.

It is proposed CFD numerical models as an alternative to subsurface or multiphase flow in porous media modeling. Such solutions can predict unstable displacements emulating flow patterns such as VF in great detail. Moreover, they can achieve remarkable accuracy on quantitative variables of interest, such as oil recovery in the Oil \& Gas industry. These numerical models could be further implemented in other fields interested in subsurface modeling such as $\mathrm{CO}_{2}$ sequestration or geothermal energy. 


\section{Acknowledgments}

The authors gratefully acknowledge Frontera Energy for having supported this research. The authors also the Information and Technology Department of the University of Los Andes (DSIT) for supplying the hardware to this research.

\section{References}

[1] R. Lenormand and C. Zarcone, "Invasion Percolation in an Etched Network: Measurement of a Fractal Dimension," Phys. Rev. Lett., vol. 54, no. 20, pp. 2226-2229, May 1985, doi: 10.1103/PhysRevLett.54.2226.

[2] R. Lenormand, E. Touboul, and C. Zarcone, "Numerical models and experiments on immiscible displacements in porous media," J. Fluid Mech., vol. 189, pp. 165-187, Apr. 1988, doi: 10.1017/S0022112088000953.

[3] M. Muskat, The Flow of Homogeneous Fluids Through Porous Media. McGraw-Hill Book Company, Incorporated, 1937.

[4] M. Muskat, Physical principles of oil production. McGraw-Hill Book Co., 1949.

[5] S. E. Buckley and M. C. Leverett, "Mechanism of Fluid Displacement in Sands," Trans. AIME, vol. 146, no. 01, pp. 107-116, Dec. 1942, doi: 10.2118/942107-G.

[6] W. F. Engelberts and L. J. Klinkenberg, "Laboratory Experiments on the Displacement of Oil by Water from Packs of Granular Material," presented at the 3rd World Petroleum Congress, Jan. 1951. Accessed: Jul. 13, 2020. [Online]. Available: https://www.onepetro.org/conference-paper/WPC-4138

[7] S. Hill and F. Inst. P, "Channeling in packed columns," Chem. Eng. Sci., vol. 1, no. 6, pp. 247-253, Jan. 1952, doi: 10.1016/0009-2509(52)87017-4.

[8] A. A. Daneshy, "Selection and Execution Criteria for Water-Control Treatments," presented at the SPE International Symposium and Exhibition on Formation Damage Control, Jan. 2006. doi: 10.2118/98059-MS.

[9] A. R. Brandt, "Oil Depletion and the Energy Efficiency of Oil Production: The Case of California," Sustainability, vol. 3, no. 10, Art. no. 10, Oct. 2011, doi: 10.3390/su3101833.

[10] J. Nittmann, G. Daccord, and H. E. Stanley, "Fractal growth viscous fingers: quantitative characterization of a fluid instability phenomenon," Nature, vol. 314, no. 6007, Art. no. 6007, Mar. 1985, doi: 10.1038/314141a0.

[11] J. Nittmann, G. Daccord, and H. E. Stanley, "WHEN ARE VISCOUS FINGERS FRACTAL?," in Fractals in Physics, L. Pietronero and E. Tosatti, Eds. Amsterdam: Elsevier, 1986, pp. 193-202. doi: 10.1016/B978-0-444-86995-1.500391.

[12] L. Paterson, "Diffusion-Limited Aggregation and Two-Fluid Displacements in Porous Media," Phys. Rev. Lett., vol. 52, no. 18, pp. 1621-1624, Apr. 1984, doi: 10.1103/PhysRevLett.52.1621.

[13] S. Tang and Z. Wei, "Laboratory experiment and numerical simulation on viscous fingering," Commun. Nonlinear Sci. Numer. Simul., vol. 1, no. 2, pp. 11-15, Apr. 1996, doi: 10.1016/S1007-5704(96)90028-3.

[14] W. Tian and D. Lu, "The Simulation of Viscous Fingering by Diffusion-Limited-Aggregation Model Based on Pressure-Oriented Rule,” Jul. 2015, pp. 1452-1455. doi: 10.2991/icaees-15.2015.271.

[15] B. Dong, Y. Y. Yan, W. Li, and Y. Song, "Lattice Boltzmann simulation of viscous fingering phenomenon of immiscible fluids displacement in a channel," Comput. Fluids, vol. 39, no. 5, pp. 768-779, May 2010, doi: 10.1016/j.compfluid.2009.12.005.

[16] B. Dong, Y. Y. Yan, and W. Z. Li, "LBM Simulation of Viscous Fingering Phenomenon in Immiscible Displacement of Two Fluids in Porous Media," Transp. Porous Media, vol. 88, no. 2, pp. 293-314, Jun. 2011, doi: 10.1007/s11242011-9740-y.

[17] Y. Shi and G. H. Tang, "Simulation of Newtonian and non-Newtonian rheology behavior of viscous fingering in channels by the lattice Boltzmann method," Comput. Math. Appl., vol. 68, no. 10, pp. 1279-1291, Nov. 2014, doi: 10.1016/j.camwa.2014.08.024.

[18] M. R. Soltanian, M. Amooie, N. Gershenzon, Z. Dai and R. Ritzi., "Dissolution Trapping of Carbon Dioxide in Heterogeneous Aquifers," Environ. Sci. Technol., vol. 51, no. 13, pp. 7732-7741, Jul. 2017, doi: 10.1021/acs.est.7b01540. 
[19] A. E. Kampitsis, A. Adam, P. Salinas, C. C. Pain, A. H. Muggeridge, and M. D. Jackson, "Dynamic adaptive mesh optimisation for immiscible viscous fingering," Comput. Geosci., vol. 24, no. 3, pp. 1221-1237, Jun. 2020, doi: 10.1007/s10596-020-09938-5.

[20] S. Lee and M. F. Wheeler, "Adaptive enriched Galerkin methods for miscible displacement problems with entropy residual stabilization," J. Comput. Phys., vol. 331, pp. 19-37, Feb. 2017, doi: 10.1016/j.jcp.2016.10.072.

[21] A. Pinilla, L. Ramirez, M. Asuaje, and N. Ratkovich, "Modelling of 3D viscous fingering: Influence of the mesh on coreflood experiments," Fuel, p. 119441, Oct. 2020, doi: 10.1016/j.fuel.2020.119441.

[22] S. Doorwar and K. K. Mohanty, "Viscous Fingering During Non-Thermal Heavy Oil Recovery," presented at the SPE Annual Technical Conference and Exhibition, Oct. 2011. doi: 10.2118/146841-MS. 